Revista lus et Praxis, Año 17, No 2, 2011, pp. 305 - 330

ISSN 0717 - 2877

Universidad de Talca - Facultad de Ciencias Jurídicas y Sociales

"El papel de la coacción en la teoría de las reglas de Hart"

Andrés Molina Ochoa

\title{
EL PAPEL DE LA COACCIÓN EN LA TEORÍA DE LAS REGLAS DE HART*
}

\author{
THE ROLE OF COERCION IN HART'S THEORY OF RULES
}

\begin{abstract}
Andrés Molina OCHOA**
\section{RESUMEN}

El objetivo del artículo es ofrecer una nueva interpretación sobre el papel de la coacción en la teoría de las reglas de H. L. A. Hart. En primer lugar, se explica por qué una inadecuada interpretación de la coerción rinde imposible la distinción entre reglas primarias secundarias y primarias, central en el pensamiento de Hart. En segundo lugar, se distingue entre coerción y reproche social, además se analiza el carácter epistemológico de El Concepto de derecho. Con base en estas dos precisiones, se sugiere que el papel de la coacción en las reglas secundarias es determinar cuándo estamos en presencia de una regla. Finalmente, se proponen posibles líneas de investigación y de solución de controversias en torno a la teoría de Hart, según las tesis defendidas en el artículo.

ABSTRACT

The objective of this article is to offer a new interpretation of the role of coercion in Hart's theory of rules. Firstly, it is discussed why an inaccurate interpretation of coercion makes impossible the important distinction between primary and secondary rules drawn by Hart. Secondly, it is analyzed the epistemological character of Hart's theory and the difference between coercion and social complains. Bases on these two clarifications, it is suggested that the role of coercion in secondary rules is to determine when we are in presence of a rule. Finally, some research lines and the solution to some controversies, following the main theses defended in this article, are suggested.

PALABRAS CLAVE Reglas, Coerción, Hart

KEY WORDS

Rules, Coercion, Hart
\end{abstract}

\footnotetext{
* El autor quiere agradecer el apoyo académico y emocional que sus estudiantes de la Facultad de Jurisprudencia de la Universidad del Rosario en Bogotá, Colombia, le brindaron durante el período comprendido entre el segundo semestre de 2008 y diciembre de 2010. Sin su ayuda, este artículo sería imposible. Además, salvo que se mencione lo contrario, todas las traducciones son del autor. Trabajo recibido el 23 de abril y aprobado el 15 de junio de 2011.

** Candidato a Doctor, Facultad de Filosofía, Universidad de Binghamton, Nueva York. Correo electrónico: andres.molina.1974@gmail.com.
} 
Aunque mucho se ha escrito sobre el pensamiento del filósofo británico ${ }^{1}$ H. L. A. Hart, poco se ha discutido el papel que tiene la coerción en su teoría jurídica, especialmente en relación con las características que diferencian a las reglas secundarias de las primarias. El problema es esencial para la correcta comprensión de su obra por dos razones; la primera está en que, como advierte el mismo Hart, "la mayor parte de las características del derecho que se han presentado como más desconcertantes y que han provocado, y hecho fracasar, la búsqueda de una definición, pueden ser clarificadas mejor si entendemos estos dos tipos de reglas y la acción recíproca entre ellos". ${ }^{2}$ Si el elemento coacción hace imposible la distinción entre reglas primarias y secundarias, la tesis central de El concepto de derecho carecería de sentido.

Una segunda razón se encuentra en la intención de Hart de desplazar a la coacción como eje a partir del cual gira la descripción del derecho. Esta pretensión se puede observar desde sus críticas a la noción del derecho como un conjunto de órdenes respaldadas por amenazas defendida por Austin, ${ }^{3}$ hasta los comentarios en contra de la tesis kelseniana según la cual sólo son reglas aquellas órdenes que imponen una sanción. ${ }^{4}$ Comentando la perspectiva de el mal hombre, desde la cual debe estudiarse el derecho según O. W. Holmes, Hart manifestó:

"¿Por qué el derecho no habría de preocuparse igual, si no más, del hombre desconcertado o del hombre ignorante que está dispuesto a hacer lo que

\footnotetext{
${ }^{1}$ La importancia de su teoría ha sido reconocida, incluso por varios de sus contradictores. Según Dworkin, por ejemplo: "He elegido esta posición [la de Hart], no solo por su claridad y elegancia sino porque aquí, como en todas partes en la filosofía del derecho, el pensamiento constructivo debe empezar con una consideración de sus puntos de vista". DwORKIN, Ronald, "The model of rules I", en Taking rights seriously, Harvard University Press, Cambridge, Estados Unidos, 1977, p. 17."[Hart] introdujo no solo los métodos de la filosofía contemporánea, en especial la filosofía del lenguaje, sino un rigor particular, uno distinguido por una admirable claridad. Esa claridad es todavía una señal de la buena jurisprudencia inglesa". GUEST, Stephen, Ronald Dworkin, Stanford University Press, Stanford, California, Estados Unidos, 1991, p. 3. Muchos autores consideran que Hart ha hecho la más importante contribución en la historia de la filosofía jurídica analítica. En este sentido, Leslie Green, por ejemplo sostiene que: "hay un consenso sobre la forma en que El concepto de derecho cambió la dirección de la teoría legal anglosajona. Entre otros motivos, porque introdujo y clarificó un conjunto de preguntas que llegaron a dominar la literatura sobre la materia: ¿Es el derecho siempre coercitivo? ¿Qué son las reglas legales? ¿Tienen los jueces discreción? [...] Hart, además acuñó el lenguaje en el cual se debaten las respuestas a estas preguntas". Ver Green, Leslie. "Philosophy and law: the concept of law revisited", Michigan Law Review 94, 1996, p. 1687. De igual forma, Kent Greenwalt califica a Hart como "[...] el más influyente positivista moderno en el mundo angloparlante". Greenwalt, Kent, "Too thin and too rich", en The autonomy of law, Clarendon Press, Oxford, Reino Unido, 1996, p. 3-4.

${ }^{2}$ HARt, H. L. A. El Concepto de Derecho, Traducción de Carrió, Genaro, Abeledo-Perrot, Buenos Aires, Argentina, 1963. p. 102. Excepto cuando se mencioné lo contrario, en este artículo se utilizará la traducción de Genaro Carrió de El concepto de derecho.

${ }^{3} \mathrm{H}_{\mathrm{ART}}$, El Concepto, cit. nota n. 2, capítulos II-IV.

${ }^{4} \mathrm{H}$ ART, El Concepto, cit. nota n. 2, pp. 45 y ss.
} 
corresponde, con tal que se le diga en qué consiste? ¿O del hombre que quiere arreglar sus asuntos, con tal de que se le diga cómo hacerlo? Por supuesto que para comprender el derecho es muy importante ver cómo lo administran los tribunales cuando llega el momento de aplicar sus sanciones. Pero esto no puede llevarnos a pensar que todo cuando hay que comprender es lo que pasa en los tribunales. Las principales funciones del derecho como medio de control social no han de ser vistas en los litigios privados o en las causas penales, que representan provisiones vitales, pero no obstante ello accesorias, para las fallas del sistema". ${ }^{5}$

Hart puede tener razón en intentar explorar otras funciones del derecho distintas a la coacción, no obstante, su teoría es inválida si no logra dar cuenta del papel de la coerción en todo ordenamiento jurídico. Es muy difícil justificar una descripción del derecho en la que no haya cabida para el tipo de sanciones y castigos que usualmente sufren quienes infringen las normas jurídicas.

El objetivo de este escrito es presentar una interpretación caritativa de la obra de Hart que permita explicar el papel de la coacción en su teoría de las reglas. El artículo está dividido en tres secciones. En la primera, se expondrá por qué la coerción, tal como es entendida por la mayoría de los autores, rinde imposible la distinción entre reglas primarias y secundarias. En la segunda parte, se analizará el carácter epistemológico de la teoría de Hart, así como se distinguirá entre reproche y coacción. Finalmente, con base en las aclaraciones hechas en la segunda parte, se sugerirá una solución al problema de la coacción y se analizarán algunas consecuencias de la interpretación defendida en este ensayo.

Por último, es importante aclarar que durante el ensayo se utilizan indistintamente las expresiones regla y norma. Aunque la teoría jurídica contemporánea con la palabra norma se refiere no sólo a las reglas sino a otro tipo de estándares como los principios y las políticas, por razones estilísticas se usan en este escrito como expresiones sinónimas.

\section{LA COACCIÓN Y LA TEORÍA DE LAS REGLAS DE HART}

En El concepto de Derecho, Hart ofrece dos criterios para diferenciar las reglas primarias de las secundarias. ${ }^{6}$ El primero se refiere al tipo de conducta que regulan ambos tipos de normas; mientras las reglas primarias " $[\ldots]$ se refieren a acciones que implican movimiento o cambios físicos; las del segundo tipo prevén actos que conducen no simplemente a movimiento o cambio físico, sino

\footnotetext{
${ }^{5} \mathrm{H}_{\mathrm{ART}}$, El Concepto, cit. nota n. 2, p. 50.

${ }^{6}$ En realidad, estos criterios ya habían sido desarrollados por Hart con anterioridad a El concepto de derecho. Ver, por ejemplo, HART, H. L.A, "Positivism and the separation of law and morals", en Essays in jurisprudence and philosophy, Oxford Clarendon Press, Oxford, Reino Unido, 1983, pp. 60-62.
} 
a la creación o modificación de deberes u obligaciones". ${ }^{7}$ El segundo criterio se refiere a la función social de cada clase de reglas; el objetivo de las reglas primarias es imponer deberes, el de las secundarias es conferir potestades. ${ }^{8}$

Según Hart, cada clase de reglas secundarias cumple la función de resolver un problema que se presentaría en una sociedad con sólo normas primarias. Si sólo existen reglas que imponen deberes, las normas se vuelven estáticas, incapaces de adecuarse a las metamorfosis que puede estar sufriendo la sociedad. Las reglas de cambio solucionan esta dificultad al introducir un procedimiento que permite la transformación y extinción deliberada de las reglas primarias. ${ }^{9}$ En general, las normas constitucionales que regulan la modificación y creación de leyes son ejemplos de reglas secundarias de cambio.

En una sociedad con sólo reglas primarias, no existe un procedimiento que autorice a una persona, o a un grupo de personas, a decidir las diferentes disputas que se presentan sobre la aplicación de las normas. Las reglas de adjudicación ${ }^{10}$ solucionan este problema al permitir a los jueces definir si las normas han sido quebrantadas y al otorgarle a la policía la potestad de asegurar el cumplimiento de las reglas primarias. Finalmente, la creciente complejidad de la sociedad y la proliferación de normas que ella genera hace necesaria la existencia de una regla que permita determinar cuáles reglas hacen parte del ordenamiento jurídico y cuáles no. Este problema, Ilamado por Hart falta de certeza, ${ }^{11}$ se resuelve mediante una regla de reconocimiento que especifica los procedimientos necesarios y suficientes para saber si una norma es o no parte del sistema.

\section{Coacción y Reglas Secundarias}

Según Hart, la reacción negativa que enfrentan quienes violan una norma es uno de los elementos que diferencian las reglas de los hábitos.Es fácil entender

\footnotetext{
${ }^{7}$ HART, El Concepto, cit. nota n. 2, p. 101.

${ }^{8} \mathrm{H}_{\mathrm{ART}}$, El Concepto, cit. nota n. 2, p. 101. Desde la publicación de la primera reseña de El concepto de derecho y del primer libro dedicado a la teoría jurídica de Hart, se cuestionaron los criterios propuestos por Hart para diferenciar las reglas primarias y secundarias. Ver, en especial: COHEN, Jonathan. "The concept of law. By H. L. A. Hart", Mind, (71)283, 1962, pp. 395-412. HACKER, P. M. S. "Hart's philosophy of law", en Law, morality and society, Clarendon Press, Oxford, Reino Unido, 1977, pp. 1-26. Responder o comentar estas críticas está por fuera del marco general de este artículo, que es, únicamente, discutir el papel de la coacción en la distinción entre reglas primarias y secundarias.

${ }^{9}$ HART, El Concepto, cit. nota n. 2, p. 115.

${ }^{10} \mathrm{H}$ ART, El Concepto, cit. nota n. 2, p. 114.

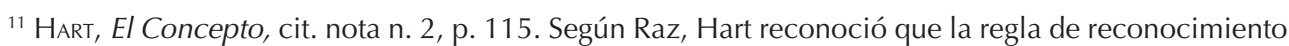
era en realidad una regla primaria. Sobre el tema, ver: RAZ, Joseph, The concept of a legal system, Oxford University Press, Oxford, Reino Unido, 1980, p. 199. SHAPIRO, Scott, "What is the rule of recognition (and does it exist)?" en The rule of recognition and the U.S. Constitution, Oxford University Press, Oxford, Reino Unido, 2009, p. 240.
} 
esta distinción ampliando un ejemplo suyo sugerido en El concepto de derecho. ${ }^{12}$ Supóngase que una persona decide acabar con un viejo hábito de ir cada sábado y domingo al teatro más cercano a su casa, para ver el último filme. Su acción en este caso podrá generar dudas: ¿no eras tú quien iba los domingos a cine?, ¿por qué no has vuelto por el teatro?, nos haces falta; sin embargo, este no es el tipo de reproche que experimenta quien ha quebrantado una regla. Si el personaje, verbi gratia, acude al cine cada fin de semana en cumplimiento de una regla impuesta por su jefe -supóngase que es crítico de un periódico importante-, recibirá fuertes críticas cuando desee cambiar de actividad: ¡Su deber es ver las películas los sábados! ¡Está incumpliendo sus obligaciones como reportero de este diario!, por ejemplo.

Según Hart, la presión por cumplir las normas es, en este sentido, un elemento esencial de las reglas, una característica fundamental que hace que una práctica sea una norma y no un simple hábito:

"Pero tal convergencia general, o aun la identidad de conducta, no basta para constituir la existencia de una regla que exija esa conducta: cuando existe tal regla las desviaciones son generalmente consideradas como deslices o faltas susceptibles de crítica, y las amenazas de desviación chocan con una presión a favor de la conformidad, si bien las formas de crítica y de presión varían según los diferentes tipos de reglas". ${ }^{13}$

Es importante aclarar que según el párrafo anteriormente citado, la coacción es una característica propia de cualquier tipo reglas. Hart no distingue entre normas primarias o secundarias, o entre reglas morales o jurídicas, al momento de referirse a las críticas que generalmente sufrirán quienes quebrantan alguna norma. Toda regla, por el hecho de serlo, contiene un elemento de reproche social sobre aquellos que actúan en contradicción con sus disposiciones. ${ }^{14}$

De forma explícita, Hart reconoce que las normas secundarias acarrean un tipo de sanción en caso de incumplimiento. Sobre la regla de reconocimiento, Hart manifestó, "Si sólo algunos jueces actuaran por su cuenta sobre la base de que lo que la Reina en Parlamento sanciona es derecho, y no apreciaran críticamente a aquellos colegas que no respetasen esta regla de reconoci-

\footnotetext{
${ }^{12} \mathrm{H}_{\mathrm{ART}}$, El Concepto, cit. nota n. 2, p. 12,

${ }^{13}$ HART, El Concepto, cit. nota n. 2, p. 70. Con anterioridad a El concepto de derecho, Hart había desarrollado ya esta idea, ver en especial: HART, H. L. A. "Legal and moral obligation", en Essays in moral philosophy, University of Washington Press, Seattle, Estados Unidos, 1958, pp. 89 y siguientes.

${ }^{14}$ No es una exageración afirmar que esta es una interpretación canónica de la teoría de las reglas de Hart. Sus dos más influyentes intérpretes, Joseph Raz y Neil MacCormick, describen los elementos de las normas según Hart de una forma similar: Ver: MAсCоRміск, Neil, Legal Reasoning and legal theory, Clarendon Press, Oxford, Reino Unido, 1978, pp. 277 y ss. Raz, Joseph, Practical reasons and norms, Princeton University Press, Princeton, Estados Unidos, 1990, pp. 49-58.
} 
miento, la característica unidad y la continuidad del sistema jurídico habrían desaparecido". ${ }^{15}$

Pese a lo anterior, no es claro cuál es el tipo de sanción que acarrea la violación de una regla secundaria. Supóngase, por ejemplo, que el Congreso de Chile aprueba un tratado internacional sin la mayoría requerida por el artículo 50, numeral uno de la Constitución. ${ }^{16}$ Para simplificar, se puede explicar el problema de la siguiente forma:

(1) Todas las reglas, incluyendo las secundarias, generan una reacción negativa en caso de incumplimiento.

(2) $X$ es una regla secundaria que autoriza al Congreso aprobar los tratados internacionales, siempre y cuando se siga el procedimiento $Y$.

(3) Por (1) y (2), debe existir una sanción en caso de incumplimiento de $X$.

¿Qué sanción podría recibir el Congreso por aprobar una ley $P$ sin seguir $Y$ ? Una primera opción sería considerar que la inconstitucionalidad de $P$ es el reproche que sufren los senadores por violar la regla $X$. En este caso, si el Tribunal Constitucional declara que $P$ es inexequible, podría pensarse que los senadores recibieron un castigo, dado que la decisión del Alto Tribunal impidió que lograran su cometido, aprobar la ley $P$. No obstante, esta opción no es posible para Hart por, al menos, dos razones.La primera es que él mismo afirma que la nulidad, en este caso la inconstitucionalidad, de una norma no es una pena, sino una consecuencia propia de una regla secundaria: "Más absurdo aún es considerar como sanción el hecho de que una medida legislativa no llega a alcanzar el status de ley si no obtiene una mayoría necesaria. Asimilar este hecho a las sanciones del derecho penal, equivaldría a concebir las reglas de tanteo (scoring rules) de un juego como dirigidas a eliminar todas las jugadas salvo la conversión de tantos". ${ }^{17}$

\footnotetext{
${ }^{15}$ HART, El Concepto, cit. nota n. 2, p. 144. En igual sentido, cuando Hart explica cuáles condiciones deben satisfacerse para hablar de un sistema jurídico, se refiere a las reglas secundarias de la siguiente forma: "La segunda condición tiene que ser satisfecha por los funcionarios del sistema. Ellos tienen que ver en las reglas pautas o criterios comunes de conducta oficial, y apreciar críticamente como fallas las desviaciones propias y las ajenas". HART, El Concepto, cit. nota n. 2, p. 145.

16 "Son atribuciones exclusivas del Congreso: 1. Aprobar o desechar los tratados internacionales que le presentare el Presidente de la República antes de su ratificación. La aprobación de un tratado se someterá a los trámites de una ley. Las medidas que el Presidente de la República adopte o los acuerdos que celebre para el cumplimiento de un tratado en vigor no requerirán nueva aprobación del Congreso, a menos que se trate de materias propias de ley. En el mismo acuerdo aprobatorio de un tratado, podrá el Congreso autorizar al Presidente de la República a fin de que, durante la vigencia de aquél, dicte las disposiciones con fuerza de ley que estime necesarias para su cabal cumplimiento, siendo en tal caso aplicable lo dispuesto en los incisos segundo y siguientes del artículo 61, y 2 . Pronunciarse respecto del estado de sitio, de acuerdo al número 2 del artículo 40 de esta Constitución".
}

${ }^{17}$ HART, El Concepto, cit. nota n. 2, p. 44 
La segunda razón está en que si la sanción de las reglas secundarias es entendida de forma igual a las primarias, no existe diferencia alguna en la función que cumplen estas normas en la sociedad. Cuando Hart afirma que las reglas primarias imponen deberes en tanto que las secundarias confieren derechos, no sólo está prestando atención a la estructura interna de las reglas, sino al papel que ambas cumplen dentro de un ordenamiento jurídico. El objetivo fundamental de las reglas primarias es evitar que una conducta sea realizada, hacer que la gente se abstenga de comportamientos que se consideran perjudiciales para la comunidad; $;^{18}$ el de las secundarias es establecer los procedimientos necesarios para producir cierto tipo de efectos jurídicos. Siguiendo un ejemplo propuesto por Hart, la función de las reglas del fútbol sobre qué es un gol, no es desalentar todas las acciones que no terminan en una anotación, sino definir cuál es el objetivo mismo del juego. ${ }^{19}$ En igual sentido, el objetivo de las reglas sobre los tratados internacionales no es impedir que sean aprobados con una mayoría distinta a la exigida, sino definir los requisitos para que esta aprobación sea válida.

Ahora bien, es posible que la diferencia entre reglas primarias y secundarias hecha por Hart esté equivocada. Lo que se pretende en este acápite no es defender la clasificación propuesta en El concepto de derecho, sino indicar que si entendemos la consecuencia de una regla secundaria como una sanción en contra del Congreso, en el caso en estudio, la taxonomía defendida por Hart deja de ser coherente con su propia teoría. Si la inconstitucionalidad de una regla es una sanción en contra de quienes crearon la norma, entonces las reglas secundarias tendrían la misma estructura y función que las primarias.

Otra posibilidad para explicar la coacción en las reglas secundarias esté en describir, estilo Kelsen ${ }^{20}$ a algunos artículos de la constitución como fragmentos de otras reglas que sí imponen sanciones. De acuerdo a esta interpretación, por ejemplo, en el artículo del Código Penal que ordena castigar a quienes cometen el delito de homicidio está contenida toda la normativa constitucional sobre la creación y modificación de las leyes. Cuando en un ordenamiento jurídico se

18 "En el caso de una regla del derecho penal podemos identificar y distinguir dos cosas: un cierto tipo de conducta que la regla prohíbe, y una sanción destinada a desalentar dicha conducta. Pero ¿cómo podríamos ver a esta luz actividades socialmente deseables tales como la que cumplen hombres cuando se intercambian promesas que no satisfacen las exigencias legales en cuanto a la forma? Esto, a diferencia de la conducta desalentada por el derecho penal, no es algo que las reglas jurídicas que establecen formalidades para los contratos estén dirigidas a suprimir. Simplemente dichas reglas no le acuerdan reconocimiento jurídico". HART, El Concepto, cit. nota n. 2, p. 43.

${ }^{19}$ HART, El Concepto, cit. nota n. 2, p. 43.

${ }^{20}$ Ver: KeLSEN, Hans, Teoría pura del derecho, $2^{\text {a }}$ Edición, Universidad Autónoma de México, México, 1982, pp. 153 y ss. Una buena descripción de la teoría de las normas de Kelsen puede encontrarse en WALTER, Robert, La estructura del orden jurídico: disertación jurídico-teórica basada en la teoría pura del derecho, Editorial Temis, Bogotá, Colombia, 1984, capítulo I. 
encuentra la regla $Z$, El que mate incurrirá en prisión, en ella se debe entender integrados los artículos de la Constitución que permiten al Congreso crear $Z$. Así las cosas, la norma completa sería, Si el Congreso crea una regla siguiendo los procedimientos establecidos en la Constitución, que ordena incurrir en prisión a quien mate, entonces quien mate incurrirá en prisión. En este sentido, se puede apreciar que las reglas secundarias propiamente no son sancionadas, su consecuencia está dada cuando la integramos con una regla primaria, con una norma que sí impone una sanción específica.

Más allá de la discusión sobre si el modelo kelseniano es más adecuado que el de El concepto de derecho, ${ }^{21}$ es claro que esta respuesta contradice el pensamiento de Hart, no sólo porque el establecer un solo tipo de normas hace imposible cualquier distinción entre las reglas jurídicas, sino porque niega la existencia de coacción en las reglas secundarias. Consciente de este problema, Hart dedicó varias páginas en El concepto de derecho a criticar y cuestionar la teoría de las normas de Kelsen. ${ }^{22}$

Finalmente, es posible pensar que la norma constitucional es en realidad coaccionada cuando una persona, verbi gratia, es sancionada por lograr de forma fraudulenta la aprobación de un tratado. Supóngase que una ley es aprobada sin los requisitos mínimos exigidos por la Constitución, porque una persona mediante actos ilícitos alteró el número de congresistas que asistieron a las sesiones en que se debatió el proyecto. En este caso, se estaría vulnerando las reglas secundarias que autorizan al Congreso a crear leyes según un procedimiento específico, esta violación acarrearía un castigo, el que sufriría quien alteró el quórum respectivo.

El problema con esta interpretación es que en realidad no explica cuál es la sanción para quien conculca las reglas secundarias. La persona del ejemplo es sancionada no por ir en contravía de los artículos de la Constitución que reglamentan la creación de las leyes, sino porque su conducta es una violación de las normas penales sobre el soborno. Por esta razón, en aquellos eventos en

\footnotetext{
${ }^{21}$ Es muy significativo, sin embargo, que muchos seguidores de la Teoría Pura del Derecho consideren equivocada la tesis kelseniana respecto a las normas facultativas. Como afirma Kucsko-Stadlmayer, al seguir la teoría de Kelsen sobre las normas: "[d]e una parte, no se expresan claramente las diferentes funciones normativas autorización y ordenación; de otra parte, en ese caso la regla de producción [la regla constitucional] sólo recibe significación cuando se puede producir sobre su base una norma coactiva y efectivamente se produce". Ver: KuCSKO-StadlmaYer, Gabriele, "Concepto y clases de normas jurídicas", en Problemas centrales de la teoría pura del derecho, Ediciones Universidad Externado de Colombia, Bogotá, Colombia, 2001, p. 51.

${ }^{22}$ Ver: HART, El Concepto, cit. nota n. 2, pp. 45 y ss. Críticas en igual sentido también fueron desarrolladas en: HART, H. L. A., "Kelsen visited", en Essays in jurisprudence and philosophy, Oxford Clarendon Press, Oxford, Reino Unido, 1983, pp. 90 y siguientes; HART, H. L. A., "Kelsen doctrine of the unitiy of law", en Essays in jurisprudence and philosophy, Oxford Clarendon Press, Oxford, Reino Unido, 1983, pp. 330 y siguientes.
} 
los cuales gracias a un error humano y no a un delito, el Congreso aprobara una ley sin el quórum necesario, no podríamos hablar de sanción en las reglas secundarias. Por lo demás, al igual que la explicación kelseniana de las normas, esta explicación hace que sea en las normas primarias donde se encuentre la sanción de las secundarias, lo cual rinde imposible la distinción entre estos tipos de reglas.

La anterior reflexión nos lleva a un grave problema para la teoría de Hart: o bien optamos por explicaciones a la Kelsen de las normas, y en este caso aceptamos que no existe una distinción entre reglas primarias y secundarias; o afirmamos que las normas secundarias no tienen sanción, lo cual hace imposible la distinción entre hábitos y reglas propuesta en El concepto de derecho.

\section{CoACCIÓN Y SANCIÓN EN LA TEORÍA de LAS NORMAS DE HART}

Para solucionar el problema de la coacción en las reglas secundarias de Hart, es necesario hacer dos precisiones importantes. La primera se refiere a por qué la sanción es una característica esencial de las reglas, la segunda a qué tipo de teoría Hart desarrolla en El concepto de derecho.

\section{La coacción y el reproche}

Aunque Hart no hace esta distinción de forma explícita, es claro que en su teoría es diferente la sanción como una reprobación social institucionalizada por el derecho, al reproche que sufre una persona por violar cualquier tipo de norma. A la primera, a partir de ahora coacción, Hart se refiere cuando describe el papel de las reglas de adjudicación," "[los ordenamientos jurídicos] han complementado las reglas primarias de obligación mediante reglas secundarias adicionales que especifican, o por lo menos limitan, los castigos por la transgresión de aquéllas, y han conferido a los jueces que verifican el hecho de la violación el poder exclusivo de disponer la aplicación de penas por otros funcionarios". ${ }^{23}$

Nótese que en este fragmento, Hart sugiere que la función de las reglas de adjudicación es institucionalizar la sanción por la violación de los deberes establecidos por normas primarias. La obligación de conducir a cierta velocidad determinada por una regla que prohíbe manejar a más de cien kilómetros por hora, es, según lo expuesto, complementada por la potestad conferida a una autoridad, la policía de tránsito, de multar a quienes quebrantan la norma. En este sentido, si existe una regla (h) que impone un deber $p$, quienes hacen $\sim p$ deberán sufrir la coacción $C$, de manos de la autoridad competente $T$, de acuerdo a la regla de adjudicación $A$.C, por tanto, es el reproche social, coacción según

${ }^{23}$ HART, El Concepto, cit. nota n. 2, p. 141. 
la terminología propuesta, que debe padecer quien vulnera (h), aunque $T$ sólo puede imponer $C$, porque $A$ así lo define.

Algo distinto es el reproche que sufren quienes violan una norma, bien sea primaria, secundaria o, incluso, moral. Es cierto que Hart de forma expresa aceptó la posibilidad de que las reglas de adjudicación, en particular, sean coaccionadas mediante reglas que imponen deberes, ${ }^{24}$ mas en este caso el castigo proviene de la violación de las reglas primarias, no de las secundarias. En otras palabras, siguiendo el ejemplo planteado, es claro que $C$ es la sanción por la violación de un deber $p$ impuesto por un regla primaria; lo que no podemos responder es cuál es el reproche social que sufre quien vulnere $A$. Aún más, dado que $A$, por definición, confiere un poder y no establece una obligación, surge la duda sobre cuál es la conducta cuya transgresión genera un reproche social. Si $T$ decide no imponer $C$, su acción será castigada por el derecho únicamente si existe una regla primaria que establece para $T$, la obligación de $C$. En este caso, $T$ será sancionado, mas no por vulnerar $A$, sino por transgredir una norma primaria.

El anterior análisis es necesario, si Hart quiere defender al mismo tiempo la tesis (i) quienes transgreden una regla encuentran una reacción negativa, y (ii) la diferencia propuesta entre normas primarias y secundarias. Como ya se discutió al inicio del artículo, Hart no puede afirmar que el reproche por la violación de las normas secundarias proviene de las primarias sin contradecirse y sin hacer que su taxonomía carezca de sentido. Por esta razón, es importante distinguir entre la coacción, como una sanción centralizada contra quienes violan los deberes impuestos por reglas primarias, y el reproche que sufren quienes vulneran cualquier tipo de reglas. En qué consiste éste para el caso de las normas secundarias es la pregunta que se pretende contestar en este escrito.

\section{El carácter epistemológico}

Uno de los aspectos más importantes y a la vez más desapercibidos en la teoría de Hart es lo que podría denominarse la finalidad epistemológica de su investigación. Contrario a lo que sus más influyentes estudiosos han afirmado, ${ }^{25}$ la pregunta que se hace Hart en El Concepto de Derecho, no es sobre qué son las reglas, sino sobre qué criterios nos permiten afirmar que una evidencia es

24 "Al igual que las otras reglas secundarias, están en un nivel diferente respecto de las reglas primarias: aunque pueden ser reforzadas mediante reglas que imponen a los jueces el deber de juzgar, ellas no imponen deberes sino que confieren potestades judiciales y acuerdan un status especial a las declaraciones judiciales relativas a la transgresión de obligaciones". HART, El Concepto, cit. nota n. 2, p. 120.

${ }^{25}$ Este problema ha pasado desapercibido no sólo por su más destacado crítico, Ronald Dworkin, sino por algunos de sus más influyentes seguidores. Ver, por ejemplo: DworkIN, The model, cit. nota n. 1. pp. 14-46. Maccormick, Neil, H. L. A. Hart, Stanford University Press, Stanford, Estados Unidos, 2008, pp. 42-60. Raz, Practical, cit. nota n. 14. 
necesaria y suficiente para decir que existe una regla. La diferencia entre los dos tipos de proyectos puede entenderse a través de la distinción que hace Searle entre tres tipos de preguntas: la ontológica indaga por lo que es el objeto de la investigación, en este caso, qué son las reglas. La causalidad pregunta qué hace un determinado objeto; en tanto que la epistemología se preocupa por cómo sabemos acerca de algo, es decir, cómo sabemos que una regla existe. ${ }^{26}$

El carácter epistemológico de la teoría de las reglas de Hart puede apreciarse con claridad si se presta atención especial a dos elementos esenciales de $E I$ concepto de derecho; el primero es la afirmación según la cual el libro hace parte de la sociología descriptiva, ${ }^{27}$ el segundo es la descripción del aspecto interno de las normas. Como se explicará a continuación, ambos sólo pueden entenderse si se parte del supuesto que Hart está desarrollando una teoría epistemológica en su obra.

La mayoría de las críticas contemporáneas al pensamiento de Hart se dirigen a su pretensión metodología de hacer una teoría neutral del derecho; ${ }^{28}$ no obstante, pocos se preguntan qué es aquello que Hart intenta describir. ${ }^{29}$ De forma más específica, qué es aquello que pretende describir a través de la filosofía y no de otras disciplinas como la sociología, por ejemplo.

Para los fundadores de la tradición filosófica a la cual se adscribe Hart, el positivismo jurídico inglés nacido a partir de los escritos de Austin y Bentham,

\footnotetext{
${ }^{26}$ Searle usa el siguiente ejemplo para ilustrar la distinción: "[...] en el caso del corazón, la ontología es qué es ese largo tejido muscular en la cavidad torácica; la epistemología es que nosotros lo descubrimos mediante el uso de estetoscopios, electrocardiogramas, si nos apuran podemos abrir el tórax y mirar allí; la causalidad es que el corazón bombea sangre a través del cuerpo". SEARLE, John, The rediscovery of the mind, The MIT Press, Cambridge, Massachusetts, Estados Unidos, 1992, p. 18.

27 "A pesar de su preocupación por el análisis, el libro puede también ser considerado un ensayo de sociología descriptiva; [...]". HART, El Concepto, cit. nota n. 2, p. 11.

${ }^{28}$ Para una introducción del debate metodológico contemporáneo, ver en especial: LeITER, Brian, "Beyond the Hart/Dworkin debate: The methodology problema in jurisprudence", American Journal of Jurisprudence, 48, pp. 17-51. PIEL, Dan. "Description and evaluation in jurisprudence", Law and Philosophy (30) 2, 2010, pp. 633-667. Molina OchOA, Andrés. "La intrascendencia del debate entre positivismo y iusnaturalismo: las normas de ius cogens", Revista Estudios Socio-jurídicos, (11)2, pp. 229-256. Para muchos autores, la principal contribución de Dworkin a la teoría jurídica está en su crítica a la metodología propuesta por Hart, lo que se ha denominado arquimedeanismo. Sobre el particular, Arthur Ripstein afirma: "El más significativo y más central tema en el trabajo de Dworkin es su rechazo a cualquier intento de investigar las preguntas en filosofía moral, legal o política desde una perspectiva ajena a la forma en que pensamos sobre ella". Ver: RiPSTEIN, Anthony, "Introduction: Anti-Archimedeanism", en Ronald Dworkin, Cambridge University Press, Nueva York, Estados Unidos, 2007, p. 5.

${ }^{29}$ Una importantísima excepción es DWORKIN, Ronald, "Hart's Postscript and the point of political philosophy", The Belknap Press of Harvard University Press, Cambridge, Estados Unidos, 2006, pp. 140-186. Aunque no lo hace de forma directa a Hart, Dan Piel pregunta a teorías que comparten su pretensión de hacer una descripción neutral de la naturaleza del derecho: "Si son descriptivas, ¿qué es eso que describen?", PIEL, "Description", cit. nota n. 28, p. 641.
} 
no existía el problema de justificar la perspectiva filosófica en la empresa de describir el derecho, puesto que para la época no había otras disciplinas que pudieran realizar esta labor. El primer departamento de sociología fue creado en 1895 por Emile Durkheim en la Universidad de Bordeaux, ${ }^{30}$ años después de la muerte de los padres del positivismo. Con el desarrollo actual de las ciencias sociales, a diferencia de sus predecesores, Hart sí tiene que explicar en qué sentido su proyecto netamente filosófico pretende ayudar o contribuir a la descripción hecha por la sociología.

La respuesta a este problema se encuentra en otras dos influencias grandes en la teoría de Hart, la obra del realista escandinavo Alf Ross y el pensamiento del filósofo analítico inglés, Peter Winch. ${ }^{31}$ Ambos autores señalaron que la descripción de ciertas instituciones humanas sólo es posible si se tienen en cuenta los conceptos y categorías que la sociedad utiliza respecto a ellas.

En su texto, La idea de una ciencia social y su relación con la filosofía, Peter Winch afirmó que las "[...] relaciones sociales son expresiones de ideas acerca de la realidad". ${ }^{32}$ Sin entender estas ideas es, por tanto, imposible una adecuada comprensión de la realidad. Supóngase que asistimos a ver la película $E I$ gran silencio, ${ }^{33}$ el casi mudo filme sobre la vida de un monasterio de la orden Cartuja en Francia. Si no sabemos qué es el catolicismo o qué es la religión, las acciones de los monjes de levantarse temprano en la mañana, asistir a misa o leer viejos libros carecen de sentido. Sin la perspectiva interna de los frailes, sin lo que ellos buscan o pretenden con esas prácticas, la descripción de su vida sería incompleta.Como afirma Winch, cualquier descripción de las prácticas de los clérigos debe "[...] tener en cuenta las ideas religiosas alrededor de las cuales la vida de los monjes gira". ${ }^{34}$

Al igual que Winch, Ross afirma que un método introspectivo es necesario para realizar una descripción de las reglas, en este caso del ajedrez:

"Uno podría tal vez pensar acercarse al problema desde una perspectiva conductista -limitándose sólo a aquello que pueda ser establecido por una observación externa de las acciones y luego encontrando algunas regularidades. No obstante, en esta forma un entendimiento de las reglas del juego nunca sería

\footnotetext{
${ }^{30}$ Ver: LuKES, Steven, Emile Durkheim: His life and work: a historical and critical study, Stanford University Press, Stanford, Estados Unidos, 1985, pp. 99 y ss.

${ }^{31}$ Sobre la influencia de Winch en el desarrollo de las teorías de las reglas de Hart, ver MACCORMICK, Legal, cit. nota n. 15, p. 278-9.

${ }^{32} \mathrm{~W}_{\mathrm{INCH}}$, Peter, The idea of social science and its relation to philosophy, Routledge \& Kegan Paul, Londres, Reino Unido, 1958, p. 23.

${ }^{33}$ GrÖNING, Philip, El gran silencio, Zeitgeist Films, Alemania, 2002, 162 minutos.

${ }^{34} \mathrm{WINCH}_{\mathrm{IN}}$ The idea, cit. nota n. 32, p. 23.
} 
alcanzado. Nunca sería posible distinguir las reglas propias del ajedrez, de una costumbre, o aun de regularidades condicionadas por la teoría del juego".$^{35}$

Siguiendo a Ross en el ejemplo del ajedrez y a Winch en el de la vida del monasterio, Hart sugiere que una perspectiva interna es necesaria para una correcta descripción de las reglas. Si sólo nos enfocamos en la apariencia seríamos incapaces de distinguir una norma de un hábito. En este sentido, Hart entiende su proyecto como filosófico y sociológico al mismo tiempo. Como la sociología no tiene la metodología requerida para describir esta perspectiva, la labor se le deja a la filosofía en su labor, como afirma el teórico del lenguaje John Austin, de completar los sobrantes de otras disciplinas. ${ }^{36}$

Hart definió el punto de vista interno como aquel de "[...] quienes no se limitan a registrar y predecir la conducta que se adecua a las reglas, sino que usan las reglas como criterios o pautas para valorar su conducta y la de los demás" ${ }^{37}$ A pesar de la importancia que tiene esta noción en el pensamiento de Hart, es una definición extraña. De hecho, dice más por lo que calla o niega que por lo que afirma.

Lo primero que niega Hart es que el punto de vista interno sea una posición moral estilo Finnis. ${ }^{38}$ De forma expresa, Hart manifestó que

"[...] algunas reglas pueden ser aceptadas simplemente por deferencia a la tradición o por el deseo de identificarse con los otros o en la creencia de que la sociedad conoce mejor qué es lo que le conviene a los individuos. [...] cuando surge la cuestión de por qué lo han hecho así los que han aceptado las reglas convencionales como guías para su comportamiento o como estándares de crítica, no veo ninguna razón para seleccionar, entre las muchas respuestas que dar, la creencia en la justificación moral de las reglas como la única respuesta posible o adecuada". ${ }^{39}$

\footnotetext{
${ }^{35}$ Ross, Alf, On law and justice, University of California Press, Berkeley, Estados Unidos, 1999, p. 13. 36 "[...] El tema de la filosofía es que ella trabaja con lo que sobra, con todos los problemas que permanecen todavía insolubles después de haber ensayado todos los otros métodos". Austin, John Langshay. "Performative-Constative". en Philosophy and ordinary language, University of Illinois Press, Illinois, Estados Unidos, 1963, p. 23

${ }^{37}$ HART, El Concepto, cit. nota n. 2, p. 122.

${ }^{38}$ Finnis sostiene que "Si hay un punto de vista en el cual las instituciones del estado de derecho, y el cumplimiento de las reglas y principios de derecho según su tenor, son considerados como, al menos presuntamente, requisitos de la racionalidad práctica, tal punto de vista es el punto de vista que debe usarse como estándar de referencia para el teórico que está describiendo las características de un orden legal". FINNIS, John, Natural law and natural rights, Oxford University Press, Oxford, Reino Unido, 1980, p. 15.

${ }^{39}$ HART, H. L. A, Post scríptum al concepto del derecho, Universidad Nacional Autónoma de México, México D.F., México, 2000, pp. 35-36. Para esta obra, utilizo la traducción de Rolando Tamayo y Salmorán.
} 
Así que usar las reglas para valorar otras acciones, según Hart, no es igual a juzgarlas desde una perspectiva moral. Un juez nazi, por ejemplo, puede seguir reglas ordenadas por Hitler, incluso juzgar a quienes las quebrantan -es ilegal el matrimonio con quienes no son arios, verbi gratia- aunque esté convencido que la normas por él aplicadas son inmorales.

La perspectiva interna tampoco es un sentimiento o una compulsión para seguir una regla, como afirmaba Ross. A lo largo de El Concepto de Derecho, Hart, de forma enfática ${ }^{40}$, sostiene que "[las experiencias sicológicas análogas a la experiencia de restricción o compulsión] no son necesarias ni suficientes para la existencia de reglas obligatorias. No es contradictorio decir que la gente acepta ciertas reglas pero no experimenta tales sentimientos de compulsión". ${ }^{41}$

¿Qué es entonces la perspectiva interna, si no es un sentimiento, un hábito o una convicción moral? En realidad, para Hart dicho punto de vista es y no es todas esas cosas. Es una posición moral, porque quien usa las reglas para valorar su conducta y la de los demás, lo puede hacer con base en sus creencias éticas. En este sentido, por ejemplo, quien conduce a la velocidad estipulada por la ley, usa esta norma básica para valorar sus acciones, pero los motivos para hacerlo pueden venir de sus convicciones morales, de su deseo por respetar la vida. ${ }^{42}$ Es una compulsión porque una persona puede seguir la misma regla de tránsito por miedo a una multa, o porque siente algún tipo de apremio debido a un accidente que sufrió en el pasado. No obstante, la perspectiva interna no es ni un sentimiento, ni una posición moral porque ellas no son necesarias para la existencia de una regla.

En otras palabras, las críticas que Hart formula tanto a Ross como a aquellas versiones iusnaturalistas del aspecto interno, se fundamenta en que es posible usar y seguir reglas sin sentir la compulsión o la posición moral de la cual hablan ellos. Es precisamente en este sentido que la teoría de Hart es epistemológica. Lo que él pretende es ofrecer otro tipo de indicativos, distintos a la moral o al sentimiento de compulsión, que nos proporcionen evidencias necesarias y suficientes ${ }^{43}$ de la existencia de reglas.

En la primera ocasión en la que Hart se refiere al aspecto interno de las reglas al inicio de El concepto de derecho, ${ }^{44}$ lo hace para distinguirlas de los hábitos.

\footnotetext{
${ }^{40}$ Ver, HART, El Concepto, cit. nota n. 2, pp: 72, 109, 172 y 308. En igual sentido, ver: HART, H. L. A., "Scandinavian realism", en Essays in jurisprudence and philosophy, Oxford University Press, Oxford, Reino Unido, 1983, pp. 161-170.

${ }^{41}$ HART, El Concepto, cit. nota n. 2, p. 83.

${ }^{42}$ Para una interpretación similar de este ejemplo, ver: EDMUNDSON, William A. "Political authority, moral powers and the intrinsic value of obedience", Oxford Journal of Legal Studies, (30) 1, pp. 187 y ss.

${ }^{43}$ Ver supra n. 37.

${ }^{44}$ HART, El Concepto, cit. nota n. 2, p. 83.
} 
Según Hart, esta característica está ya contenida en las otras diferencias que previamente ha señalado entre reglas y hábitos, no obstante, por su importancia y porque no es tenida en cuenta por varios teóricos del derecho tiene que ser explicada con más detenimiento. ${ }^{45}$ Con lo anterior, Hart parece querer decir que el aspecto interno de las reglas se encuentra en que (i) "[...] la desviación del curso regular [de la regla] suscite alguna forma de crítica"46 y (ii) en que dicha crítica sea "[...] considerada como legítima o justificada". ${ }^{47}$

No obstante, en posteriores ocasiones, Hart de forma enfática sostiene que tanto (i) como (ii) son expresiones o manifestaciones del aspecto interno de la reglas. ${ }^{48}$ Dicho de otro modo, dado que el aspecto interno se viste de múltiples ropajes -una convicción moral, según Finnis, o una compulsión, estilo Rossnos es imposible determinar en realidad qué es; no obstante, somos capaces de determinar que existe cuando se presentan (i) y (ii); es decir, cuando tenemos la evidencia necesaria y suficiente para afirmar que estamos en presencia de una regla. ${ }^{49}$

La teoría de las reglas de Hart, en este sentido, se asemeja a tres teorías importantes en la historia de la filosofía..$^{50}$ La primera es la solución ofrecida por Descartes al problema de las otras mentes. Aunque es imposible conocer a ciencia cierta si otros seres humanos tienen una mente como la nuestra, po-

\footnotetext{
${ }^{45}$ HART, El Concepto, cit. nota n. 2, p. 70.

${ }^{46} \mathrm{H}_{\mathrm{ART}}$, El Concepto, cit. nota n. 2, p. 70.

${ }^{47}$ HART, El Concepto, cit. nota n. 2, p. 70.

${ }^{48}$ Hart utiliza dos verbos, manifest y display, para referirse a (i) y (ii) en relación con el aspecto interno de las reglas: "[...] they are internal statements in the sense that they manifest acceptance of the standards and use and appeal to them in various ways". HART, "Scandinavian", cit. nota n. 38 p. 167. "These views are manifested in the criticisms of others and demands for conformity upon others when deviation is actual or threatened, and in the legitimacy of such criticism and demands when received from others. [...] What is necessary is that there should be a critical reflective attitude to certain pattersn of behavour as a common standard, and that this should display itself in criticism". HART, H. L. A. The concept of law, Clarendon Press, Oxford, Gran Bretaña, 1994, p. 57.
}

${ }^{49}$ Es asombrosa la poca atención entre los estudiosos de Hart a que (i) y (ii) en realidad son manifestaciones del aspecto interno de las reglas y no otros dos elementos diferentes de las reglas. Incluso, un autor tan cuidadoso y tan conocedor de la teoría de Hart como MacCormick sólo propone esta tesis de forma dubitativa: "No es totalmente claro si tales expresiones de demandas y críticas son concebidas como constitutivas, o meramente evidencias, de la actitud crítica reflexiva concebida (sic)", MAcCormick, Legal, cit. nota n. 14, p. 275. Por lo demás, es claro que Hart no puede considerar las expresiones y críticas como constitutivas del aspecto interno de las reglas, dado que son claramente prácticas externas.

${ }^{50}$ Las posiciones epistemológicas tampoco son extrañas dentro de la teoría jurídica. Explicando la costumbre, por ejemplo, Celano Bruno sostiene: "Según la noción de costumbre aquí acogida, el comportamiento repetido, que constituye el aspecto exterior de la costumbre, deberá entenderse como expresión de una actitud subjetiva (la acción deberá interpretarse como signo, y, también, como indicio, o prueba, de una intención". Ver: Bruno, Celano, "Dos estudios sobre la costumbre", en Biblioteca de ética, filosofía del derecho y política, Editorial Fontamara, México D.F., 2000, p. 46. 
demos diseñar un test o una prueba que nos justifique en el juicio que afirme que otra persona tiene una mente. Si el otro se comparta como uno, actúa como uno, se está justificado en adscribirle el mismo tipo de experiencia que se tiene cuando uno se comporta de forma semejante. Incluso si no se sabe a ciencia cierta qué es una mente, o qué estado interior tiene el otro, esto no constituye un serio problema, porque, de igual forma, estamos justificados en afirmar, dado su comportamiento externo, que él tiene experiencias similares a las nuestras. ${ }^{51}$ Siguiendo a Descartes, Géraud de Cordemoy diseñó pruebas para responder a la pregunta sobre si alguien tenía o no una mente. ${ }^{52}$ En ellas, se establecía que si una persona expresaba e interpretaba las experiencias al igual que las hace quien práctica el experimento, entonces era irracional no atribuirle otra mente.

La segunda es la prueba ofrecida por Chomsky sobre la posibilidad de afirmar que una persona sigue reglas. Para él, esto sólo es viable, porque el otro parece ser una persona que exhibe características de voluntad, uso del lenguaje y otras indicaciones de la inteligencia. ${ }^{53}$ Estos comportamientos no son propiamente seguir una regla, pero ellos son la evidencia necesaria y suficiente para determinar si alguien es capaz o no de seguir normas.

Otro ejemplo de doctrinas epistemológicas es el famoso test de Turing, ${ }^{54}$ diseñado para probar si una máquina es capaz de pensar. Turing describió un complejo test que debería satisfacerse para afirmar de forma justificada que una máquina actuó de forma inteligente. Una vez más, el objetivo de la teoría no es definir qué es la inteligencia, sino establecer un método que permita probar cuando ésta existe. ${ }^{55}$

Tanto Descartes, Chomsky, Turing o Hart desarrollan teorías epistemológicas. Ellos ofrecen una serie de evidencias necesarias y suficientes para probar la existencia de algo sobre lo cual no tenemos un acceso directo: las reglas, la mente o la inteligencia. En el caso de Hart, su teoría es epistemológica dado

\footnotetext{
${ }^{51}$ DeSCARTES, René, Meditaciones acerca de la filosofía primera: seguidas de las objeciones y respuestas, Universidad Nacional de Colombia, Bogotá, Colombia, 2010. Para un estudio sobre la teoría de Descartes sobre las otras mentes, ver: Hoffman, Paul. "Descarte's theory of distinction", Philosophy and phenomenological research, (64)1, 2002, pp. 57-78.

${ }^{52}$ Ver: CHOMsky, Noam, Language and thought, Moyer Bell, Wakefield, Rhode Island, Estados Unidos, 1993, pp. 36 y siguientes.

${ }^{53}$ С номsку, Noam, Knowledge of language: its nature, origin, and use, Praeger, Nueva York, Estados Unidos, 1986, p. 235.

${ }^{54}$ TURING, Alan, "Computering machinery and intelligence", Mind, (59)235, 1950, pp. 433-460. Para una discusión contemporánea del test de Turing, ver: Hernández-Orallo, José, "Beyond the Turing test", Journal of Logic, Language, and Information, 9, 2000, pp. 447-466.

${ }^{55}$ Para la relación entre el test de Turing y la teoría cartesiana de otras mentes, ver: CHOMskr, Language, cit. nota n. 52, p. 38.
} 
que sugiere que toda norma se caracteriza porque es considerada por al menos algunos agentes desde un aspecto interno, el cual aunque imposible de definir, sí se puede comprobar mediante las manifestaciones de críticas justificadas ante quienes violan las reglas. Estas críticas no son el aspecto interno, ni siquiera son características de las reglas, son sólo la evidencia necesaria para probar que estamos en presencia de una norma. ${ }^{56}$

\section{LA SOLUCIÓN}

A lo largo del artículo, se han explicado dos tesis contradictorias defendidas por Hart: (i) Todas las reglas, incluyendo las secundarias, generan una reacción negativa en caso de incumplimiento, y (ii) No existe un reproche social en caso de violación de una norma secundaria.Para solucionar esta aparente contradicción es necesario prestar atención a la ubicación del fragmento en $E I$ concepto de derecho en que Hart desarrolla su teoría de las reglas. ${ }^{57}$ Lo que de forma mayoritaria se conoce como teoría de las reglas de Hart ${ }^{58}$ es, de hecho, una pequeña sección de un capítulo en el cual él pretende probar que el modelo de ordenamiento jurídico sugerido por Austin es incapaz de explicar la continuidad en el derecho.

En la medida en que Hart desarrolla su teoría jurídica, su teoría de las reglas se va sofisticando y cambiando. Cuando Hart explica el papel de las normas secundarias y cómo estas significan el paso de una sociedad pre legal a una jurídica, afirma:

"[b]ajo el régimen simple de las reglas primarias el punto de vista interno se manifiesta, en su forma más sencilla, en el uso de aquellas reglas como fundamento para la crítica, y como justificación de las exigencias de conformidad, presión social y castigo [...] Con el agregado de las reglas secundarias,

\footnotetext{
${ }^{56}$ No es esta la única vez en que Hart utilizó eventos externos para probar estados internos. En uno de sus primeros artículos publicado con el filósofo inglés Stuart Hampshire, Hart se pregunta por la evidencia externa necesaria para probar que una persona ha tomado una decisión. Ver: HAMPSHIRE, Stuart y HART, H. L. A. "Decision, intention and certainty", Mind 67, 1958, pp.1-12. En igual sentido, al Hart explicar cuando se está justificado en afirmar que un contrato existe, sugiere: "[...] dadas las dificultades de la prueba o como una cuestión de política social, puede en ocasiones adoptar lo que hemos Ilamado estándares objetivos o externos, los cuales tratan ciertas formas de comportamiento exterior como evidencia concluyente de la existencia de un estado mental [...]" HART, H. L. A. "Problems of the philosophy of law", en Essays in jurisprudence and philosophy, Oxford Clarendon Press, Oxford, Reino Unido, 1983, p. 96.

${ }^{57}$ Ver: HART, El Concepto, cit. nota n. 2, p. 55 y siguientes.

${ }^{58}$ Por sólo citar unos ejemplos, se fundamentan en esta sección para comentar la teoría de las reglas de Hart: Raz, Practical, cit. nota n. 14, pp 49-58. Dworkin, "The Model", cit. nota n. 1, pp. 17 y siguientes. MacCormick, Legal, cit. nota n. 15, pp. 257 y siguientes. AleXANDER, Larry; SHerwin, Emily, The rule of rules, Duke University Press, Durham, Estados Unidos, 2001, pp. 20 y siguientes. SHAPIRO, Scott. "What is the internal point of view", Faculty Scholarship Series, 1336, 2006, pp. 1-22.
} 
el campo de lo que se hace y dice desde el punto de vista interno se extiende y diversifica mucho". ${ }^{59}$

En otras palabras, la evidencia que había sugerido al inicio del libro para probar la existencia del aspecto interno cambia, se transforma en la medida en que las reglas secundarias aparecen en la sociedad. En estas circunstancias, la posición interna no sólo se manifiesta o expresa a través de la presión social sobre aquellos que han violado las normas, sino a través de enunciados como: El derecho dispone que. ${ }^{60}$

De acuerdo a Hart, a partir del surgimiento de la regla de reconocimiento, ella determina el mecanismo, o los procedimientos necesarios para probar que las otras normas son válidas, para identificar que existen. ${ }^{61}$ Para verificar la existencia de una regla, una vez estamos en frente de un ordenamiento jurídiCo, no es necesario señalar las manifestaciones del aspecto interno de la regla - las críticas en casos de vulneraciones a la norma, por ejemplo- sino que basta con acudir, en palabras de Hart, "[...] a una sanción legislativ a; a la práctica consuetudinaria; a las declaraciones generales de las personas especificadas; o a las decisiones judiciales pasadas, dictadas en casos particulares" ${ }^{\prime 62}$

Una vez una sociedad ha dado el paso de una comunidad pre legal, con sólo reglas primarias, a un sistema jurídico, el reproche social no es necesario para probar la existencia de una norma. Este avance no significa que no se necesite un sistema centralizado de coacción que garantice la eficacia requerida para la existencia del ordenamiento jurídico. De hecho, sucede lo contrario, con el paso del tiempo, los sistemas jurídicos advierten "[...] las ventajas de una centralización adicional de la presión social; y han prohibido parcialmente el uso de castigos físicos o de auto-ayuda violenta por los particulares" ${ }^{63}$

Así las cosas, la aparición de un ordenamiento jurídico hace que por un lado la coacción se centralice a través de reglas secundarias de adjudicación que definen el procedimiento y la forma en que serán castigados quienes violan las normas primarias; por otro lado, permite que la represión social deje de ser necesaria para probar la existencia de las reglas primarias. Una vez se abandona el estadio pre legal, la sociedad dispone de un sistema propio para la identificación de los estándares que hacen parte del ordenamiento jurídico, la regla de reconocimiento.

\footnotetext{
${ }^{59} \mathrm{H}_{\mathrm{ART}}$, El Concepto, cit. nota n. 2, p. 122.

${ }^{60} \mathrm{H}$ ART, El Concepto, cit. nota n. 2, p. 128.

${ }^{61}$ "Dondequiera se acepte tal regla de reconocimiento, tanto los particulares como los funcionarios tienen criterios con autoridad para identificar las reglas primarias de obligación". HART, El Concepto, cit. nota n. 2, p. 125.

${ }^{62} \mathrm{H}_{\mathrm{ART}}$, El Concepto, cit. nota n. 2, p. 126.

${ }^{63} \mathrm{H}_{\mathrm{ART}}$, El Concepto, cit. nota n. 2, p. 121.
} 
En realidad, Hart está sólo elaborando una teoría a partir de lo que podría llamarse el entendimiento común del mundo del derecho. ${ }^{64}$ Cuando dos personas discuten sobre la existencia o no de una ley, la solución está dada a partir de una serie de procedimientos tales como acudir a la publicación oficial de la norma y la concordancia o no de la ley con un texto constitucional. La idea de Hart era que todos estos mecanismos hacían parte de una regla de reconocimiento la cual, a diferencia de la norma básica de Kelsen, podría ser probada sociológicamente. ${ }^{65}$

Ahora bien, es imposible verificar la existencia de la regla de reconocimiento a través de los procedimientos por ella misma establecidos. La prueba de que los jueces utilizan una regla como último criterio de identificación de otras normas, no puede ser la regla misma, al igual que aplicar el test de tuberculina a los médicos no sirve para probar que los galenos la utilizan para el diagnóstico de la tuberculosis. Una cosa es diagnosticar una enfermedad -probar la existencia de la norma primaria-, otra muy distinta verificar que se emplea un método para hacer un diagnóstico -utilizar la regla de reconocimiento.

Para probar la regla de reconocimiento, Hart acude entonces al mismo procedimiento que había planteado para verificar la existencia de cualquier norma en ausencia de un ordenamiento jurídico; es decir, a que se presente una práctica cuya vulneración genera una crítica generalizada. Al igual que en las sociedades pre legales, es posible determinar la existencia del aspecto interno de una norma a partir de las críticas hechas a quienes las quebrantan, en el caso de la regla de reconocimiento, la aceptación de los jueces se determina porque ellos aprecian "[...] críticamente tales desviaciones como fallas frente a los criterios vigentes, que son esencialmente comunes y públicos" ${ }^{\prime 6}{ }^{66}$

La solución al problema planteado aparece clara entonces, el reproche social de las reglas secundarias consiste en apreciar críticamente las acciones de aquellos jueces que no siguen lo facultado por la regla de reconocimiento. La función de la sanción también queda clara, no es evitar que una norma sea vulnerada, sino el método sugerido por Hart para probar la existencia de la regla de reconocimiento.

\footnotetext{
${ }^{64}$ Recuérdese que El concepto de derecho se inspiró en el asombro que le produjeron a Hart afirmaciones tan extrañas a lo que el común de la gente entiende por derecho, como las hechas por Holmes y el ala más extrema del realismo jurídico norteamericano, quienes negaban la existencia de las reglas jurídicas. Ver: HART, El Concepto, cit. nota n. 2, primer capítulo. Para una descripción de los motivos que Hart para escribir El concepto de derecho, ver: LACEY, Nicola, A life of H. L. A. Hart: the nightmare and the noble dream. Oxford University Press, Oxford, Reino Unido, 2004.

${ }^{65}$ Sobre la relación entre la regla de reconocimiento de Hart y la norma básica de Kelsen, ver RAmOS PASCUA, José Antonio, La regla de reconocimiento en la teoría jurídica de H. L.A. Hart, Tecnos, Madrid, España, 1989, pp. 30 y siguientes. PAulson, Stanley, Fundamentación crítica de la doctrina de Hans Kelsen, Universidad Externado de Colombia, Bogotá, Colombia, 2000, pp. 77 y siguientes.

${ }^{66} \mathrm{H}$ ART, El Concepto, cit. nota n. 2, p. 144.
} 
Si la descripción de la teoría de Hart hecha es correcta, sólo se ha resuelto de forma parcial el problema de la coacción en las reglas secundarias. Aunque se ha explicado por qué es necesaria y en qué consiste la sanción en el caso de la regla de reconocimiento, no se ha solucionado el problema en el caso las reglas secundarias de adjudicación y cambio.

Existe una importante diferencia entre las reglas secundarias y primarias de la cual Hart habla poco a lo largo de El concepto de derecho, pero que es esencial para entender el papel de la coacción en su obra. Para la existencia de un ordenamiento jurídico, no es necesario que se acepten las reglas primarias, únicamente basta con que sean obedecidas. ${ }^{67}$ En el caso de las reglas secundarias, en cambio, es necesario probar la aceptación de las normas por parte de los funcionarios oficiales. ${ }^{68}$

Como se señaló en el artículo, dado que es imposible acceder de forma directa a la mente de los funcionarios judiciales, es necesario diseñar una prueba que especifique las condiciones mínimas y necesarias para afirmar que una regla secundaria es aceptada. Las críticas a quienes vulneran las normas es, según Hart, uno de los elementos necesarios para probar que existe este elemento interno.

El reproche social, en este sentido, cumple una función epistemológica respecto de las reglas secundarias en la obra de Hart. No es, como podría pensarse, una sanción jurídica, sino las críticas y expresiones condenatorias de quienes no siguen lo estipulado por las normas secundarias. Gracias a estas manifestaciones, es posible afirmar que existe una regla de reconocimiento y que los oficiales han aceptado las reglas de adjudicación y de cambio, elementos esenciales para probar se está en presencia de un ordenamiento jurídico.

\section{ConClusión}

En el artículo se ha pretendido demostrar que el reproche social es necesario para probar la existencia de la regla de reconocimiento, dado que, a diferencia de las normas primarias, su pertenencia al sistema no puede verificarse apelando a los procedimientos que ella misma define. En segundo lugar, se ha sugerido que la sanción social, entendida como críticas a quienes no siguen las reglas secundarias, es fundamental para verificar que los funcionarios judiciales aceptan este tipo de normas, lo cual no es necesario en las reglas primarias, toda vez

\footnotetext{
67 "En un caso extremo el punto de vista interno con su característico uso normativo del lenguaje jurídico ('Esta es una regla válida') podría limitarse al mundo oficial". HART, El Concepto, cit. nota n. 2, p. 146.

68 "La afirmación de que un sistema jurídico existe es, por lo tanto, un enunciado bifronte, una de cuyas caras mira a la obediencia por parte de los ciudadanos ordinarios, y la otra a la aceptación de reglas secundarias como pautas o criterios comunes críticos de conducta oficial, por parte de los funcionarios". HART, El Concepto, cit. nota n. 2, p. 145.
} 
que la aceptación de los particulares no es requerida para probar la existencia de un ordenamiento jurídico.

Una de las más importantes conclusiones que se desprenden de la solución planteada está en la labor de la academia y en el de los teóricos del derecho para la preservación del sistema jurídico. Supóngase, por ejemplo, que en un país existe la regla de reconocimiento $X$ que, en palabras de Hart, unifica el sistema jurídico. Para determinar la existencia de $X$, para saber en qué consiste, es necesario prestar atención a las prácticas de los jueces y las críticas hechas cuando $X$ es vulnerada. En el caso en que los jueces, por ejemplo, decidan aplicar $X_{1}$, en lugar de $X$, las personas sólo notarán que se ha vulnerado la regla básica del ordenamiento jurídico si existen críticas a los jueces que han quebrantado la norma de reconocimiento.

Las polémicas que surgieron a partir de Plessy v. Fergusson, ${ }^{69}$ el caso que permitió la discriminación racial en los Estados Unidos, son un buen ejemplo de la importancia de la Academia, de los teóricos del derecho e, incluso de los activistas sociales, en la conservación de la regla de reconocimiento y, por tanto, de la integridad del ordenamiento jurídico. Gracias precisamente a la presión de la opinión pública, de académicos y de organizaciones sociales, la Corte Suprema de Justicia se dio cuenta del error que había cometido en Plessy v. Fergusson y enmendó su error en Brown v. Board of Education. ${ }^{70}$

Las protestas en contra de Plessy y el movimiento que dio origen al cambio jurisprudencial en Brown no buscaban cambiar la Constitución o alterar el ordenamiento jurídico; al contrario, ellas invocaban la Carta Política para enmendar el daño causado por el precedente judicial que permitió la discriminación racial y que transgredió la Constitución estadounidense. ${ }^{71}$ Estas protestas ilustran lo que quizás Hart tuvo en mente cuando sugirió que las críticas a quienes violaban una norma eran una prueba de la existencia de una regla. Sin las voces que se alzaron en contra de Plessy, los estadounidenses difícilmente se habrían dado cuenta que ésta en realidad no fue una simple sentencia, sino una violación a la regla de reconocimiento por entonces válida. ${ }^{72}$

\footnotetext{
${ }^{69}$ Homer A. Plessy v. Fergusson. Estados Unidos, Corte Suprema de Justicia, 18 de mayo de 1896.

${ }^{70}$ Oliver Brown et al. v. Board of Education of Topeka et al. Estados Unidos, Corte Suprema de Justicia, 17 de mayo de 1954.

${ }^{71}$ Ver: Brown v. Board of Education, ver: Kıuger, Richard, Simple justice, Vintage Books, Nueva York, Estados Unidos, 1977. Irons, Peter, "Give me the colored doll", en A people's history of the Supreme Court, Penguin Books, Nueva York, Estados Unidos, 2000, pp. 383-394.

72 Uno de los principales contradictores de Hart, Ronald Dworkin, sugiere una interpretación parecida a la dada en este artículo sobre el papel de la crítica para determinar el contenido de la regla de reconocimiento. Para Dworkin, la desobediencia civil se encuentra justificada, por cuanto quienes la realizan ayudan a probar la constitucionalidad de una norma, dado el contenido moral de la Carta Política estadounidense. Ahora bien, a diferencia de Hart, Dworkin sugiere que es en la deliberación
} 
El papel de la coacción en la obra de Hart también puede ayudar a contestar algunas de las críticas más importantes en contra de su obra. Si la teoría de las reglas de Hart es epistemológica, tal y como se describió en este artículo, entonces la razón no asiste a quienes la critican por no poder dar cuenta de aquellos eventos en los cuales una persona sigue una regla de forma individual. ${ }^{73}$ Aunque la discusión de estas críticas sobrepasa el tema de este escrito, una respuesta plausible a ellas podría desarrollarse a partir de distinguir la existencia de una norma y su prueba.Es posible que existan reglas que son seguidas de forma individual y que, por tanto, no generen críticas cuando han sido violadas, no obstante, no disponemos de los medios necesarios para probar su existencia.

Finalmente, es posible que las críticas de Bulygin y MacCormick ${ }^{74}$ respecto a una posible falacia de circularidad entre la regla de reconocimiento y de adjudicación pueda ser también resuelta atendiendo al papel de la coacción en las reglas secundarias. La posible circularidad se advierte cuando se señala que la regla de reconocimiento sólo puede probarse mediante la actividad de los jueces, quienes sólo existen gracias a una norma de adjudicación que les ordena aplicar las reglas señaladas por la regla de reconocimiento. Aunque discutir estas críticas también sobrepasa el tema de este artículo, es plausible pensar que una posible defensa de la teoría de Hart se pueda encontrar en el papel del reproche social, el cual, tal como se ha señalado en este artículo, no sólo puede provenir de otros jueces.

En un artículo reciente, la socióloga Margaret Gilbert afirmó: "Quizás la más influyente y conocida teoría de las reglas sociales es aquella que Hart sugirió en su ahora clásico libro $E /$ concepto de derecho".$^{75} \mathrm{~A}$ más de cincuenta años de su publicación, más que una refutación de su obra, quizás sea necesaria una adecuación de sus contenidos y un mayor esfuerzo por clarificar aquellos pasajes oscuros que han servido para difundir los errores y no las virtudes de la obra.

moral que se da en el juicio de quienes desobedecen la norma, en donde se puede examinar si la regla transgredida es o no constitucional. Lo interesante es que para ambos, las críticas a quienes se desvían de la constitución (Dworkin) o de la regla de reconocimiento (Hart) son importantes al momento de determinar el contenido de las normas. Ver: DworkIN, Ronald. "Civil disobedience", en Taking rights seriously, Harvard University Press, Cambridge, Estados Unidos, 1977, pp. 223-239.

${ }^{73}$ Tanto Raz como Dworkin sugieren que la teoría de las reglas de Hart es incapaz de explicar el caso de quien sigue una norma de forma individual, como el vegetariano que vive en una sociedad de fanáticos de comer carne. Ver: Dworkin, "The Model", cit. nota n. 2; RAz, Practical, cita nota n. 15.

${ }^{74}$ Ver en especial, Bulrain, Eugenio. "Algunas consideraciones sobre los sistemas jurídicos", Doxa 9, 1991, pp. 257-279. МасCоRмICK, H. L. A., cita nota n. 25, pp. 130 y siguientes.

${ }^{75}$ Gilbert, Margaret. "Social rules: some problems for Hart's account and alternative proposal", Law and Philosophy (18)141, 1999, p. 141. 


\section{BiBLIOGRAFÍA}

AleXANDer, Larry; Sherwin, Emily, The rule of rules, Duke University Press, Durham, Estados Unidos, 2001.

Austin, John Langshay, "Performative-Constative"en Philosophy and ordinary language, University of Illinois Press, Illinois, Estados Unidos, 1963.

Bruno, Celano, "Dos estudios sobre la costumbre", en Biblioteca de ética, filosofía del derecho y política, Editorial Fontamara, México D.F., 2000.

BulYGIN, Eugenio, "Algunas consideraciones sobre los sistemas jurídicos", Doxa 9, 1991.

Chomsky, Noam, Knowledge of language: its nature, origin, and use, Praeger, Nueva York, Estados Unidos, 1986. , Language and thought, Moyer Bell, Wakefield, Rhode Island, Estados Unidos, 1993.

Coren, Jonathan, "The concept of law. By H. L. A. Hart", Mind, (71)283, 1962.

DesCARTES, René, Meditaciones acerca de la filosofía primera: seguidas de las objeciones y respuestas, Universidad Nacional de Colombia, Bogotá, Colombia, 2010.

DworkIN, Ronald, "The model of rules I", en Taking rights seriously, Harvard University Press, Cambridge, Estados Unidos, 1977, pp. 14-46.

"Hart's Postscript and the point of political philosophy", The Belknap Press of Harvard University Press, Cambridge, Estados Unidos, 2006.

EDMUNDSON, William A. "Political authority, moral powers and the intrinsic value of obedience", Oxford Journal of Legal Studies, (30) 1.

FINNIS, John, Natural law and natural rights, Oxford University Press, Oxford, Reino Unido, 1980.

GiLBERT, Margaret, "Social rules: some problems for Hart's account and alternative proposal", Law and Philosophy (18)141, 1999.

Green, Leslie, "Philosophy and law: the concept of law revisited", Michigan Law Review, 94, 1996.

Greenwalt, Kent, "Too thin and too rich", en The autonomy of law, Clarendon Press, Oxford, Reino Unido, 1996.

GrönING, Philip, El gran silencio, Zeitgeist Films, Alemania, 2002, 162 minutos.

Guest, Stephen, Ronald Dworkin, Stanford University Press, Stanford, California, Estados Unidos, 1991.

HACKER, P. M. S., "Hart's philosophy of law", en Law, morality and society, Clarendon Press, Oxford, Reino Unido, 1977. 
HampshirE, Stuart;Hart, H. L. A. "Decision, intention and certainty", Mind 67, 1958.

HaRT, H. L. A., The concept of law, Clarendon Press, Oxford, Gran Bretaña, 1994. ,El Concepto de Derecho, Traducción de Carrió, Genaro, AbeledoPerrot, Buenos Aires, Argentina, 1963.

,"Legal and moral obligation", en Essays in moral philosophy, University of Washington Press, Seattle, Estados Unidos, 1958.

, "Positivism and the separation of law and morals", en Essays in jurisprudence and philosophy, Oxford Clarendon Press, Oxford, Reino Unido, 1983.

, "Problems of the philosophy of law", en Essays in jurisprudence and philosophy, Oxford Clarendon Press, Oxford, Reino Unido, 1983. "Kelsen visited", en Essays in jurisprudence and philosophy, Oxford Clarendon Press, Oxford, Reino Unido, 1983. , "Kelsen doctrine of the unitiy of law", en Essays in jurisprudence and philosophy, Oxford Clarendon Press, Oxford, Reino Unido, 1983. ,Post scríptum al concepto del derecho, Universidad Nacional Autónoma de México, México D.F., México, 2000.

, "Scandinavian realism", en Essays in jurisprudence and philosophy, Oxford University Press, Oxford, Reino Unido, 1983.

Hernández-Orallo, José, "Beyond the Turing test", Journal of Logic, Language, and Information, 9, 2000.

Hoffman, Paul, "Descarte's theory of distinction", Philosophy and phenomenological research, (64)1, 2002.

Homer A. Plessy v. Fergusson. Estados Unidos, Corte Suprema de Justicia, 18 de mayo de 1896.

IRONS, Peter, "Give me the colored doll", en A people's history of the Supreme Court, Penguin Books, Nueva York, Estados Unidos, 2000.

KelSEN, Hans, Teoría pura del derecho, 2ª Edición, Universidad Autónoma de México, México, 1982.

KıUger, Richard, Simple justice, Vintage Books, Nueva York, Estados Unidos, 1977.

Kucsko-Stadlmayer, Gabriele, "Concepto y clases de normas jurídicas", en Problemas centrales de la teoría pura del derecho, Ediciones Universidad Externado de Colombia, Bogotá, Colombia, 2001.

LACEY, Nicola, A life of H. L. A. Hart: the nightmare and the noble dream. Oxford University Press, Oxford, Reino Unido, 2004.

LeITER, Brian, "Beyond the Hart/Dworkin debate: The methodology problema in jurisprudence", American Journal of Jurisprudence, 48, 2003. 
LUKES, Steven, Emile Durkheim: His life and work: a historical and critical study, Stanford University Press, Stanford, Estados Unidos, 1985.

Maccormick, Neil, Legal Reasoning and legal theory, Clarendon Press, Oxford, Reino Unido, 1978.

Maccormick, Neil, H. L. A. Hart, Stanford University Press, Stanford, Estados Unidos, 2008.

Molina OCHOA, Andrés, "La intrascendencia del debate entre positivismo y iusnaturalismo: las normas de ius cogens", Revista Estudios Socio-jurídicos, (11)2.

Oliver Brown et al. v. Board of Education of Topeka et al. Estados Unidos, Corte Suprema de Justicia, 17 de mayo de 1954.

Paulson, Stanley, Fundamentación crítica de la doctrina de Hans Kelsen, Universidad Externado de Colombia, Bogotá, Colombia, 2000.

PIEL, Dan. "Description and evaluation in jurisprudence", Law and Philosophy (30) 2, 2010.

Ramos Pascua, José Antonio, La regla de reconocimiento en la teoría jurídica de H. L.A. Hart, Tecnos, Madrid, España, 1989.

Raz, Joseph, Practical reasons and norms, Princeton University Press, Princeton, Estados Unidos, 1990.

RAz, Joseph, The concept of a legal system, Oxford University Press, Oxford, Reino Unido, 1980.

RIPSTEIN, Anthony, "Introduction: Anti-Archimedeanism", en Ronald Dworkin, Cambridge University Press, Nueva York, Estados Unidos, 2007.

Ross, Alf, On law and justice, University of California Press, Berkeley, Estados Unidos, 1999.

SeARLE, John, The rediscovery of the mind, The MIT Press, Cambridge, Massachusetts, Estados Unidos, 1992.

SHAPIRO, Scott, "What is the rule of recognition (and does it exist)?" en The rule of recognition and the U.S. Constitution, Oxford University Press, Oxford, Reino Unido, 2009.

1336, 2006.

"What is the internal point of view", Faculty Scholarship Series,

TURING, Alan. "Computering machinery and intelligence", Mind, (59)235, 1950.

WALTER, Robert, La estructura del orden jurídico: disertación jurídico-teórica basada en la teoría pura del derecho, Editorial Temis, Bogotá, Colombia, 1984.

WINCH, Peter, The idea of social science and its relation to philosophy, Routledge \& Kegan Paul, Londres, Reino Unido, 1958. 
